\title{
Telephone-supported computerised
} cognitive-behavioural therapy: REEACT-2 large-scale pragmatic randomised controlled trial

Simon Gilbody, Sally Brabyn, Karina Lovell, David Kessler, Thomas Devlin, Lucy Smith, Ricardo Araya, Michael Barkham, Peter Bower, Cindy Cooper, Sarah Knowles, Elizabeth Littlewood, David A. Richards, Debbie Tallon, David White and Gillian Worthy, on behalf of the REEACT collaborative

\section{Background}

Computerised cognitive-behavioural therapy (CCBT) for depression has the potential to be efficient therapy but engagement is poor in primary care trials.

\section{Aims}

We tested the benefits of adding telephone support to CCBT

\begin{abstract}
Method
We compared telephone-facilitated cCBT (MoodGYM) $(n=187)$ to minimally supported CCBT (MoodGYM) $(n=182)$ in a pragmatic randomised trial (trial registration: ISRCTN55310481). Outcomes were depression severity (Patient Health Questionnaire (PHQ)-9), anxiety (Generalized Anxiety Disorder Questionnaire (GAD)-7) and somatoform complaints (PHQ-15) at 4 and 12 months.
\end{abstract}

\section{Results}

Use of CCBT increased by a factor of between 1.5 and 2 with telephone facilitation. At 4 months PHQ-9 scores were 1.9 points lower (95\% Cl 0.5-3.3) for telephone-supported CCBT. At 12 months, the results were no longer statistically significant (0.9 PHQ-9 points, $95 \% \mathrm{Cl}-0.5$ to 2.3). There was improvement in anxiety scores and for somatic complaints

\section{Conclusions}

Telephone facilitation of CCBT improves engagement and expedites depression improvement. The effect was small to moderate and comparable with other low-intensity psychological interventions.

\section{Declaration of interest}

None.

\section{Copyright and usage}

(c) The Royal College of Psychiatrists 2017.
Depression is the most common mental health disorder in community settings and is estimated to become the second largest cause of global disability by $2020 .^{1}$ It is one of the most common reasons for consulting with a primary care physician and its associated personal and economic burden is considerable. ${ }^{2}$ Although antidepressants remain an important treatment option, many patients and healthcare professionals would like to access psychological therapy as an alternative or adjunct to drug therapy. ${ }^{3}$ Cognitive-behavioural therapy (CBT) has emerged as a leading evidence-supported form of brief psychological therapy for people with depression. ${ }^{4}$ However, demand for CBT cannot be met from existing therapist resources. ${ }^{5}$ One promising alternative to therapist-delivered CBT is the use of self-help interventions including the provision of therapy via computer. ${ }^{6}$ In recent years a number of interactive programs have been developed that enable CBT to be delivered by computer (computerised CBT (cCBT)). If effective, such programs have the potential to expand the provision of psychological therapy in primary care and may represent an efficient and effective form of care for depression. ${ }^{7}$ In an earlier large-scale pragmatic trial (the first Randomised Evaluation of the Effectiveness and Acceptability of Computerised Therapy (REEACT) trial) ${ }^{8,9}$ we compared two commonly used cCBT packages (MoodGYM or Beating the Blues) v. usual primary care under real-world conditions to test their effectiveness (rather than efficacy). Participants were proactively offered technical support and weekly encouragement to use the computer packages, but we purposely did not augment the content of psychological therapy over the telephone. The cCBT in the first REEACT trial was therefore a form of supported self-help, but was not one that was guided by a clinician. The first REEACT trial is, at the time of writing, the largest publicly funded independently conducted primary care trial of cCBT. The main finding of the trial was that for the primary outcome of depression severity at 4 months there was no significant benefit when participants were offered technically supported cCBT in addition to usual general practitioner (GP) care. The most likely explanatory mechanism of lack of effect was poor uptake and use of computer packages by trial participants under real-world conditions. $^{9}$

Systematic reviews have highlighted the potential for CCBT to be effective but have also further demonstrated variable effect sizes and substantial between-study heterogeneity. ${ }^{10,11}$ One important source of between-study heterogeneity is the level of support that is made available to people who are offered treatment with CCBT. cCBT requires a person with depression to engage with a self-help computer-based technology. Research has shown that people with depression often do not engage with cCBT, and only a minority actually complete all of the planned sessions of the computer package. ${ }^{12}$ This observation is consistent with a broader body of research into the uptake and effectiveness across the range of self-help interventions for depression such as bibliotherapy (selftreatment using written materials). ${ }^{13}$ Research in the area of self-help treatments for depression has demonstrated that entirely self-guided materials (with no professional support) are likely to be less effective than self-help technologies where there is a level of guidance and professional support ('guided self-help'). Unsupported self-help treatment (including unsupported computer-delivered self-help) has been shown in systematic reviews to have minimal or relatively small effect sizes. ${ }^{13}$ In contrast, more intensively and professionally supported treatments have generally been found in efficacy trials to have moderate effect sizes claimed to be comparable with those 
achieved with face-to-face therapy. ${ }^{14}$ To our knowledge the comparative effectiveness of minimally supported cCBT $v$. more intensively supported cCBT has not been directly tested in large-scale, independently-conducted, head-to-head, pragmatic trials (although there are some head-to-head comparisons in smaller-scale trials ${ }^{15}$ ). We postulated on the basis of these findings, and on the basis of emerging trial-based evidence summarised in systematic reviews (for example Andersson \& Cuijpers ${ }^{10}$ ), that people with depression might engage with $\mathrm{CCBT}$ and it might show an effect, but only if offered alongside a high level of facilitation and support. We designed the present study (the REEACT-2 trial, trial registration: ISRCTN55310481) to test this hypothesis and to generate trial-based evidence on the best means of delivering CCBT in primary care mental health services.

\section{Method}

\section{Study design and patients}

The REEACT-2 trial was designed to examine the additional benefits of telephone facilitation and structured guidance alongside a free-to-use computer-delivered CBT package (MoodGYM). The comparator was minimally supported cCBT. Participants in both arms were given access to MoodGYM, an accompanying booklet, a freephone number for technical support and continued with usual GP care. MoodGYM is a free-to-use, internet-based, interactive CBT program for depression developed and copyrighted at the Australian National University Centre for Mental Health Research. The online program is accompanied by a booklet with exercises and quizzes and consists of five interactive modules released sequentially and lasting approximately 30$45 \mathrm{~min}$ and a sixth session that is predominantly consolidation and revision. Study participants were asked to complete one session each week. The program provides patients with CBT techniques to overcome patterns of unhelpful thinking using cartoon characters to represent habits of thought.

\section{Experimental arm}

Participants in the telephone-facilitated cCBT (experimental) arm were allocated a telephone support worker (TSW) who provided a programme of weekly telephone calls. The background of the TSWs was that of a graduate-level support worker. The telephone facilitation programme comprised eight telephone calls to be completed alongside the cCBT program within 14 weeks of first contact from the TSW (and before the 4-month follow-up time point). The purpose of the first and longest session (30-40 min) was to introduce the participant to the principles of CBT and the MoodGYM program and booklet, explain the process and help the participant identify difficulties and goals, and feel confident about engaging with the intervention. The following six sessions were between 10 and $20 \mathrm{~min}$ long and were intended to provide motivation and to help participants identify any barriers to engagement with CCBT and to the achievement of their goal(s). The final session helped participants to consolidate what they had learned from cCBT and discuss their next steps and, if appropriate, how they might use the MoodGYM program in the future. The telephone facilitation programme was delivered according to a manual developed by co-investigator K.L. in conjunction with the REEACT-2 team. TSWs received 1 day of training in the delivery of the intervention. Clinical supervision was given to trial TSWs by investigators K.L., D.K. and S.G.

\section{Comparator arm}

All participants in the control group were registered as users of MoodGYM and given a unique password. As with the intervention group, they were supplied with a free helpline number to ring if they had technical problems or needed advice and a booklet explaining MoodGYM, but they did not receive regular phone calls. This comparator intervention replicated UK National Health Service care in most settings and represented what would happen if a patient were given the website of a CCBT package such as MoodGYM by their GP or primary care mental health worker without being offered proactive support.

The study population comprised patients selected from primary care with depression or low mood as determined by a score of ten or more on the Patient Health Questionnaire (PHQ)-9. ${ }^{16}$ This cut-off point is known to detect clinical depression (major depression) in primary care populations ${ }^{17}$ with acceptable sensitivity and specificity. The REEACT-2 participants were recruited from a mix of rural and urban UK primary care practices in and around Bristol, Avon, Somerset, Gloucestershire, Manchester, Sheffield, Derbyshire, South Yorkshire, Humberside, East Yorkshire, Durham, Tyneside and Northumberland.

Participants meeting the following criteria were eligible to enter the study: (a) aged 18 or above; (b) not currently in receipt of cCBT or specialist psychological therapy; (c) a score of $\geqslant 10$ overall (indicating moderate, moderately severe or severe depression) and $<3$ for question 9 (measuring suicidal thoughts) on the PHQ-9 depression instrument. ${ }^{16}$ Both individuals with incident and prevalent primary care cases of depression were included. In line with the pragmatic nature of this trial, patients were eligible to participate whether or not they were in receipt of antidepressant medication or had comorbid physical illness or non-psychotic functional disorders. We excluded people currently in receipt of psychological therapy. We also excluded potential participants who: (a) were actively suicidal as identified by their GP or as reported by item 9 on the PHQ-9; (b) had been bereaved within the past year; (c) had given birth within the past year; (d) had a diagnosis of psychotic depression; (e) had a primary diagnosis of alcohol or drug misuse; (f) were not able to read and write in English.

\section{Randomisation and masking}

Simple randomisation was performed using a computer-generated random number sequence. At the end of the baseline appointment study researchers telephoned a secure randomisation line at the York Trials Unit and were given participant allocation and MoodGYM login details. Participants were informed immediately.

\section{Outcome measures}

The pre-specified primary outcome was depression severity and symptomatology as measured on a validated self-report continuous measure (PHQ-9) ${ }^{16}$ at 4 months. The secondary outcome measures were: PHQ-9 at 12 months (as a continuous measure); PHQ-9 at 4 and 12 months (dichotomous measure at cut-point PHQ-9 $\geqslant 10){ }^{16}$ anxiety (Generalised Anxiety Disorder Questionnaire (GAD-7)); ${ }^{18}$ somatoform complaints (PHQ-15); ${ }^{19}$ health-state utility (EuroQol - EQ5D); ${ }^{20}$ and service use using the adapted Client Service Receipt Inventory $(\mathrm{CSRI})^{21}$ at 4 and 12 months.

The REEACT-2 trial was powered on the basis of an ability to detect a between-group difference in PHQ scores. We sought to recruit 350 patients with depression - 175 participants per arm. The REEACT-2 trial was designed to have sufficient power to detect a Cohen's $d$ effect size of 0.30 with $80 \%$ power allowing for loss to follow-up of $20 \%$ in line with our empirically based estimates from the first REEACT trial. The final sample size for 
the two arms was 369 and we exceeded the pre-specified sample size.

\section{Statistical analysis}

All outcomes were summarised descriptively by intervention group and at each time point using mean, median, standard deviation, range and number of patients for continuous outcomes and number of patients and percentage for discrete outcomes. The primary outcome was the severity of depression as measured by the PHQ-9 as a continuous measure at 4 months. Statistical analyses were performed in SAS version 9.3.

The PHQ-9 score was summarised and analysed as a continuous outcome. This was summarised for each assessment time point (baseline, 4 and 12 months) using mean, standard deviation, median and range, and the number of missing values. Plots were presented showing the mean and 95\% confidence interval at each time point. A repeated measures mixed regression model was used to analyse the change in PHQ-9 score over time. This included all randomised participants (intention to treat) and provides reliable estimates assuming the data are missing at random. The outcome was the PHQ-9 score at 4 and 12 months and the model included the baseline PHQ-9 score, treatment group, age, gender, baseline GAD-7 score and time. The treatment $\times$ time interaction was included to evaluate if the difference between treatments changed over time. The mean difference, $95 \%$ confidence interval and $P$-values are presented for all terms in the model. Effect sizes (Cohen's $d$ ) were calculated for the between-group differences in mean PHQ-9 score at 4 and 12 months using the difference between the means and corresponding standard errors from the mixed model. The standard errors were converted to standard deviations using the corresponding sample size in each treatment group.

The dichotomous analysis (not depressed (PHQ-9 $<10$ )/ depressed (PHQ 9 $\geqslant 10$ )) compared minimally supported cCBT with telephone-facilitated cCBT using a logistic regression model adjusting for the baseline PHQ-9 score, age, gender, baseline GAD-7 score and treatment. The dichotomous analysis was on a complete case basis (only including those with a 4-month assessment). A sensitivity analysis was performed using simple imputation and a worst case scenario. This assumed that all participants with a missing outcome were still clinically depressed with a PHQ-9 score $\geqslant 10$.

The GAD-7 and the PHQ-15 scores were analysed as continuous outcomes using the same repeated measures mixed models described for PHQ-9 above. Resource use data and health state utilities (derived from the EQ5D) formed the basis of a full economic evaluation and are described in the full study report. ${ }^{22}$ Adherence by participants to the computer program was measured by requesting information from the website providing MoodGYM (hosted by the developers of MoodGYM at the Australian National University). We obtained computer usage data on the number of times each participant logged on to the MoodGYM program and whether each module was 25, 50, 75 or $100 \%$ complete. Adverse events were classified according to their seriousness and relationship to the intervention.

\section{Results}

A total of 369 participants were randomised to the two-armed comparison of minimally supported CCBT $(n=182)$ with telephone-facilitated cCBT $(n=187)$. The first participant was randomised on the 24 June 2011 and the last on the 25 April 2013. The flow of participants through the trial is shown in the CONSORT diagram (online Fig. DS1). The two groups were well balanced at baseline for gender, age, ethnicity and education. The mean age of participants was 40.6 years $($ s.d. $=13.8$ ) (online Table DS1). The study population was mostly White British (94\%) and $64.5 \%$ were women. The minimally supported cCBT and telephone-facilitated cCBT groups were balanced at baseline for employment. The majority (61.5\%) of participants were employed and of these $23.6 \%$ were absent from work because of depression at the time of their baseline assessment. The severity of depression at baseline (as ascertained by the median PHQ-9 score) was 16 (range 10-25), which corresponds with a moderate to high level of severity.

\section{PHQ-9 as a continuous outcome}

At the 4-month primary outcome the between-group difference in PHQ-9 scores was 1.9 points (95\% CI 0.5-3.3) in favour of telephone-facilitated CCBT, with a standardised effect size (Cohen's $d$ ) of $0.32(P=0.009)$ (online Table DS2). At 12 months, there was no longer evidence of a between-group difference in PHQ-9 scores $(0.9,95 \%$ CI -0.5 to 2.3$)$. Using a repeated measures analysis over the whole trial period the between-group difference in PHQ-9 scores was 1.4 (95\% CI 0.2-2.6) in favour of telephone-facilitated CCBT with a standardised effect size (Cohen's $d$ ) of $0.27(P=0.0253)$ (online Table DS2). Table 1 and Fig. 1 show the PHQ-9 scores at each assessment time point (baseline, 4 and 12 months) for both groups.

\begin{tabular}{|c|c|c|c|c|c|c|}
\hline & \multicolumn{2}{|c|}{ Baseline } & \multicolumn{2}{|c|}{ Month 4} & \multicolumn{2}{|c|}{ Month 12} \\
\hline & $\begin{array}{c}\text { Minimally } \\
\text { supported cCBT }\end{array}$ & $\begin{array}{c}\text { Telephone- } \\
\text { facilitated cCBT }\end{array}$ & $\begin{array}{c}\text { Minimally } \\
\text { supported cCBT }\end{array}$ & $\begin{array}{c}\text { Telephone- } \\
\text { facilitated cCBT }\end{array}$ & $\begin{array}{c}\text { Minimally } \\
\text { supported CCBT }\end{array}$ & $\begin{array}{c}\text { Telephone- } \\
\text { facilitated cCBT }\end{array}$ \\
\hline \multicolumn{7}{|l|}{ PHQ-9 continuous score } \\
\hline$n$ & 182 & 187 & 128 & 141 & 132 & 142 \\
\hline Mean (s.d.) & $16.4(4.1)$ & $16.8(3.9)$ & $10.4(6.4)$ & $8.5(6.3)$ & $9.2(6.2)$ & $8.2(6.4)$ \\
\hline Median (range) & $16(10-25)$ & $17(10-26)$ & $10(0-27)$ & $7(0-24)$ & $9(0-25)$ & $7(0-27)$ \\
\hline \multicolumn{7}{|l|}{ PHQ-9 dichotomised score } \\
\hline$n$ & 182 & 187 & 128 & 141 & 132 & 142 \\
\hline Depressed, $n$ (\%) & $182(100)$ & $187(100)$ & $66(51.6)$ & $51(36.2)$ & $57(43.2)$ & $46(32.4)$ \\
\hline Not depressed, $n$ (\%) & 0 & 0 & $62(48.4)$ & $90(63.8)$ & $75(56.8)$ & $96(67.6)$ \\
\hline Missing at follow-up, $n(\%)^{a}$ & 0 & 0 & $54(30)$ & $46(25)$ & $50(27)$ & $45(24)$ \\
\hline
\end{tabular}




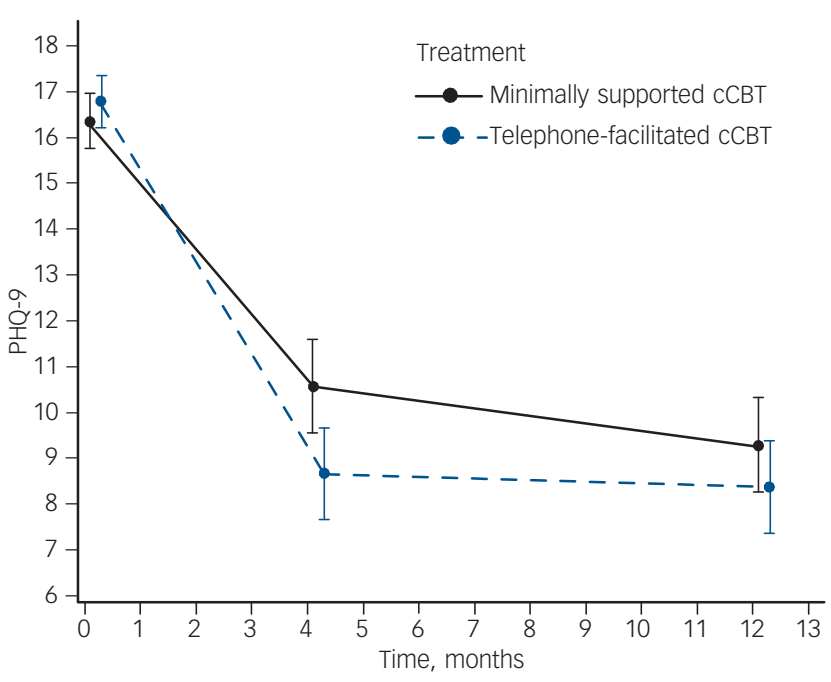

\section{Fig. 1 Depression severity at each assessment.}

Severity is mean and $95 \%$ confidence interval Patient Health Questionnaire (PHQ)-9 score. Results from a repeated measures, mixed model adjusting for baseline score, age, gender, baseline Generalized Anxiety Disorder Questionnaire (GAD)-7 score and time. CCBT, computerised cognitive-behavioural therapy.

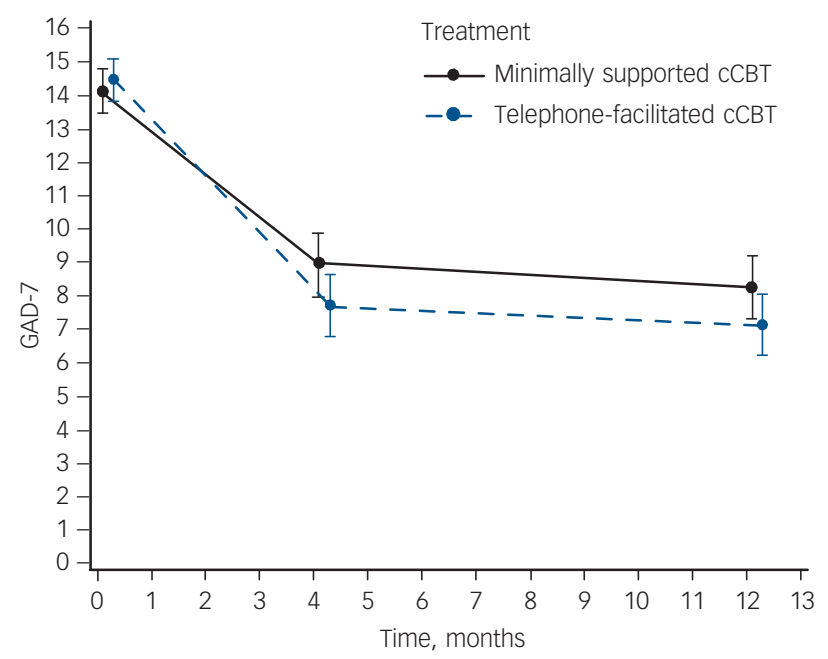

\section{Fig. 2 Anxiety severity at each assessment.}

Severity is mean and $95 \%$ confidence interval Generalized Anxiety Disorder Questionnaire (GAD)-7 score. Results from a repeated measures, mixed mode adjusting for baseline score, age, gender and time. CCBT, computerised adjusting for baseline score, age,
cognitive-behavioural therapy.

\section{PHQ-9 as a dichotomous outcome}

After 4 months $66(51.6 \%)$ of the 128 participants in the minimally supported cCBT group and $51(36.2 \%)$ of the 141 in the telephone-facilitated cCBT group had a PHQ-9 score $\geqslant 10$ (Table 1). The odds of no longer being depressed (defined as PHQ-9 <10) at 4 months were increased twofold in the telephone-facilitated cCBT group compared with minimally supported cCBT (odds ratio $(\mathrm{OR})=2.05,95 \%$ CI 1.23-3.42). The benefit of telephone-facilitated cCBT was no longer significant at 12 months $(\mathrm{OR}=1.63,95 \% \mathrm{CI} 0.98-2.71 \mathrm{P}=0.06)$.

\section{Other secondary outcomes}

For secondary outcomes there was a significant between-group difference in anxiety scores (GAD-7) in favour of telephonefacilitated cCBT when all time points were considered (between-group

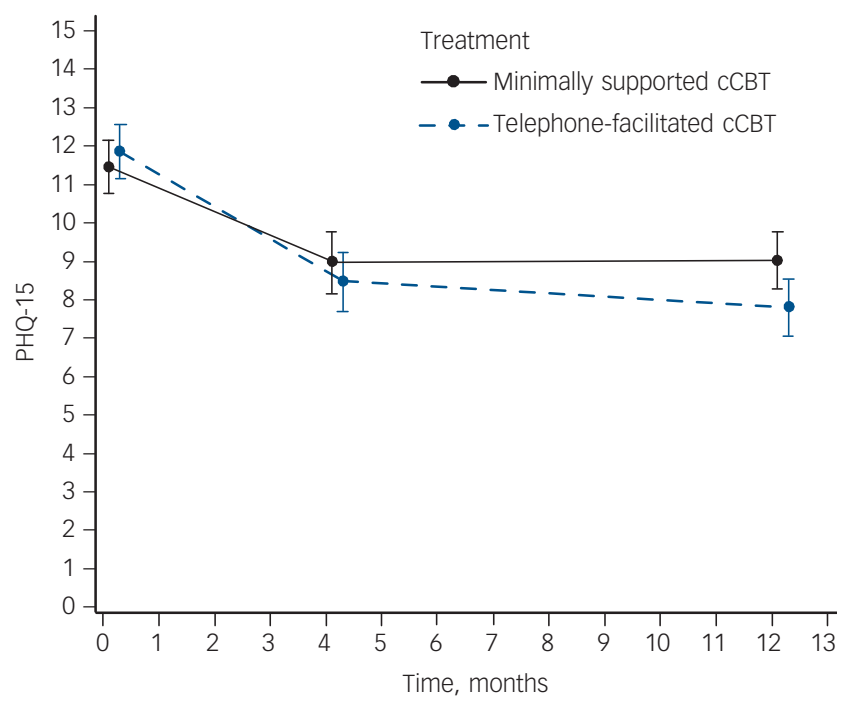

Fig. 3 Severity of somatoform complaints at each assessment.

Severity is mean and $95 \%$ confidence interval Patient Health Questionnaire (PHQ)-15 score. Results from a repeated measures, mixed model adjusting for baseline score, age, gender, baseline Generalized Anxiety Disorder Questionnaire (GAD)-7 score and time. CCBT, computerised cognitive-behavioural therapy.

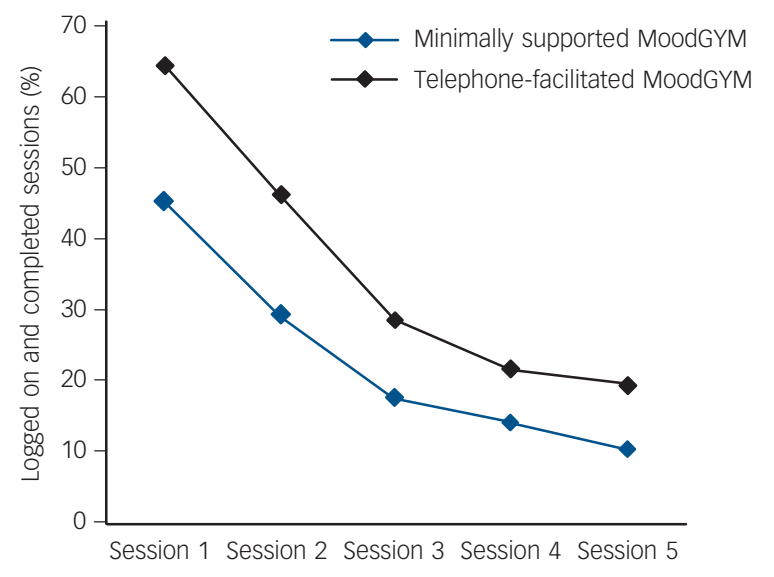

Fig. 4 MoodGYM usage, session by session, as ascertained by computer login records.

difference 1.2, 95\% CI 0.1-2.3, $P=0.037$ ) (Fig. 2 and online Table DS3). For somatic complaints there was a borderline significant difference in favour of telephone-facilitated CCBT when all time points were considered (between-group difference 1.1, 95\% CI $0.0-1.8, P=0.051$ ) (Fig. 3 and online Table DS4).

\section{Adherence and adverse events}

When computer records were scrutinised there were few participants who completed all five sessions in either minimally supported (10.4\%) or telephone-facilitated cCBT (19.4\%) (online Table DS5). Usage was generally increased by a factor of between 1.5 and 2 when telephone facilitation was offered, with $46.2 \%$ of participants in receipt of telephone facilitation completing two or more sessions $v .29 .1 \%$ of participants with minimal support (Fig. 4). There was a total of ten serious adverse events, none of which was thought to be related to the trial. All were reviewed by the Trial Steering Committee and the Data Monitoring and Ethics Committee. 


\section{Discussion}

\section{Main findings}

REEACT-2 is one of the largest trials of computer-delivered CBT to date. The trial tested whether the addition of structured telephone facilitation substantially increased engagement with computer-mediated CBT and resulted in improved outcomes. We purposely designed a pragmatic trial to test effectiveness under real-world conditions rather than efficacy under ideal but restrictive conditions in order to maximise the external validity of our results. ${ }^{23}$ The main finding of the REEACT-2 trial is that the addition of structured telephone facilitation resulted in significant reductions in depression severity compared with cCBT with technical support alone. The effect size was moderate and was most evident in the short term (4 months) and had diminished by 12 months. Telephone facilitation of cCBT therefore expedited depression improvement, although the absence of benefit at 12 months is unsurprising given the average duration of an episode of depression is less than 12 months. When depression was considered as a binary outcome, the odds of no longer being depressed were twice as high in the telephonefacilitated group at 4 months. Benefits in terms of psychological outcomes were also observed using a validated anxiety scale and for somatoform complaints when outcomes were averaged over a 12-month period. Engagement with the technology was increased through the addition of telephone facilitation.

\section{Comparison with findings from other trials}

The REEACT-2 trial drew upon a manualised form of telephone support, which can readily be delivered after a relatively brief period of training. At present computerised CBT is offered by many healthcare systems as a minimally supported low-intensity psychological intervention and as part of a stepped care framework. The intervention trialled in the REEACT-2 study therefore represents an enhancement of care that can be readily delivered at scale in primary care settings. The results of this trial should be considered alongside other trials and systematic reviews of cCBT and low-intensity interventions for common mental disorders. Our earlier study (the REEACT trial) ${ }^{8}$ was similarly a large-scale pragmatic trial of CCBT where one arm included the free-to-use cCBT package (MoodGYM). In that previous trial we offered a low-intensity form of technical telephone support and found that usage was low and there were no additional clinical benefits of cCBT when it was added to usual primary care. This led us to speculate that an enhancement in the level of support and guidance might increase uptake and effectiveness. Evidence that the addition of guidance to CCBT is associated with a greater level of effectiveness comes from systematic reviews, where pooled estimates of the effect size of trials with therapist guidance are larger than the pooled effect size obtained from unsupported cCBT. ${ }^{10}$ Evidence also comes from a systematic review of small-scale head-to-head comparisons of unsupported $v$. supported cCBT in a range of common mental disorders. ${ }^{15}$ This hypothesis has now been directly tested in the present randomised controlled trial that, to our knowledge, is the first test of this in a large-scale (adequately powered) direct randomised head-to-head comparison. The results of the REEACT 2 trial are also comparable with other primary care based psychological treatments, ${ }^{24}$ but the effect size observed in REEACT-2 is smaller compared with other developer-led trials of cCBT. ${ }^{10}$ The additional benefit of guided support is in line with the results of a systematic review of three small-scale studies in depression. ${ }^{15}$

\section{Strengths and limitations}

The REEACT-2 trial has several strengths in its design. First, this trial was pragmatic in design and recruited from primary care, whereas most trials to date have recruited from online populations or by participant advertisement. This addresses a major shortcoming of the literature identified by Andersson \& Cuijpers in their 2009 review. ${ }^{10}$ The results of REEACT-2 are therefore more generalisable to clinical populations encountered in primary care. Second, the trial was significantly larger than other trials to date (see Baumeister et $a l^{15}$ ) and had sufficient power to detect more modest effect sizes. Third, we conducted a pragmatic trial of effectiveness rather than efficacy by trialling a low-intensity enhancement to CCBT that could be delivered at distance to a range of people fulfilling very broad depression inclusion criteria (typical of those encountered in primary care). Fourth, the period of follow-up was 1 year and this allows some conclusions to be drawn about the durability of effect. Finally, we were able to study the actual use of computer technology by our trial participants with reference to computer records.

There were also limitations to the REEACT-2 study. First, in view of the pragmatic nature of the design there was loss to follow-up of around a quarter of the participants overall, and we know very little about the outcomes of these participants. Second, we did not measure outcome with a clinical interview to establish the presence of depression according to accepted classification systems. Instead we relied on self-report measures of depression severity; although these are well-validated against diagnostic systems. ${ }^{25}$ Third, even with the provision of telephone facilitation, only a small proportion of participants in either arm completed all sessions of the cCBT program. There is possibly more still that can be offered to enhance the uptake of computer therapy. Finally, the level of depression severity at baseline was moderate to high, and this is at the upper range of severity recommended in some stepped care systems. ${ }^{4}$ However, the positive results of the REEACT-2 trial provide supportive evidence that low-intensity interventions can be offered to this group and will result in improved outcomes. This finding is consistent with recent reviews of the effectiveness of low-intensity interventions across the range of severities of depression. ${ }^{26}$

\section{Implications}

The implications for practice and policy that emerge from the first REEACT and REEACT-2 trials are twofold. The first is that minimally supported cCBT results in very low levels of uptake and confers little over usual care. We would therefore suggest that healthcare systems do not offer this form of unsupported treatment as part of stepped care. However, unsupported cCBT should still be offered as a form of direct access treatment to non-clinical populations, although the benefits that might be expected are likely to be small. The second implication is that the addition of structured telephone facilitation (such as that designed in REEACT-2 to work alongside MoodGYM) will result in greater levels of engagement with computer technology. In turn this will produce moderate clinical improvements and reductions in the proportion of people who continue to experience depression over a 4 - to 12 -month period. Telephone support is a low-intensity enhancement of care that can be offered at scale and could be readily implemented in most healthcare settings as part of a stepped care system.

\section{Funding}

This project was funded by the UK NIHR Health Technology Assessment programme (project number HTA 06/43/504). The views and opinions expressed therein are those of the 
authors and do not necessarily reflect those of the HTA programme, NIHR, NHS or the Department of Health. The funder played no role in the study design, in the collection, analysis or interpretation of the data, in the writing of the paper or in the decision to submit the article for publication. All authors were independent from the funders. Data sharing: reasonable requests for patient level data should be made to the corresponding author and will be considered by the REEACT publications management group. Consent for data sharing was not obtained but the presented data are anonymised and risk of identification is low.

\section{Acknowledgements}

We would like to thank especially the patients from primary care who agreed to be recruited to take part in this trial. Thanks also to members of the Primary Care Research Network (PCRN), GPS, research nurses, administrative and other staff at participating GP practices, the Mental Health Research Network (MHRN) and the site research teams. In addition, we would like to thank the Trial Steering Committee and Data Monitoring and Ethics Committee members for overseeing the study. We thank too Gwen Brierley who was the trial manager at the start of the trial and who co-wrote the trial protocol and REC applications; Anna Thake, D.T., S.K. and D.W. Who were the trial coordinators at the
sites; the many researchers, clinical studies officers and research nurses who recruited sites; the many researchers, clinical studies officers and research nurses who recruited
participants to the study and collected data; the York Trials Unit for providing the randomisation service, for managing the data and conducting the analysis of the clinical data; and the team of telephone support workers. We would also like to extend our gratitude to the developers of MoodGYM, in particular to Kylie Bennett and Ada Tam, for providing us with participant logins and usage data.

The REEACT-2 trial is dedicated to the memory of Professor Helen Lester (1961-2013) who contributed time and wisdom at every stage of the REEACT trial programme.

Simon Gilbody, DPhil, FRCPsych, Sally Brabyn, MA, MSC, Department of Health Sciences, University of York, York; Karina Lovell, RN, MSc, PhD, School of Nursing Midwifery and Social Work, University of Manchester, Manchester; David Kessler, MBBS, MRCPsych, MD, MRCGP, Academic Unit of Primary Health Care, University of Bristol, Bristol; Thomas Devlin, PhD, Lucy Smith, BA, PGDip, Department of Health Sciences, University of York, York; Ricardo Araya, PhD, MRCPsych, Centre of Global Mental Health, London School of Hygiene and Tropical Medicine, London; Michael Barkham, PhD, Centre for Psychological Services Research, University of Sheffield, Sheffield; Peter Bower, PhD, NIHR School for Primary Care Research, of Sheffield, Sheffield; Peter Bower, PhD, NIHR School for Primary Care Research
University of Manchester, Manchester; Cindy Cooper, PhD, School of Health and Related Research, University of Sheffield and Clinical Trials Research Unit, University of Sheffield, UK; Sarah Knowles, PhD, NIHR School for Primary Care Research, University of Manchester, Manchester; Elizabeth Littlewood, PhD, Department of Health Sciences, University of York, York; David A. Richards, RN, PhDhc, PhD, University of Exeter Medical School, University of Exeter, Exeter; Debbie Tallon, MSC, School of Social and Community Medicine, University of Bristol, Bristol; David White, MPH, Clinical Trials Research Unit, University of Sheffield, Sheffield; Gillian Worthy, MSC, York Trials Unit, University of York, York, UK

Correspondence: Simon Gilbody, Department of Health Sciences, Mental Health Services Research, Department of Health Sciences, University of York, York Y010 5DD, UK. Email: simon.gilbody@york.ac.uk

First received 25 Aug 2016, final revision 29 Oct 2016, accepted 3 Nov 2016

\section{References}

1 Whiteford HA, Degenhardt L, Rehm J, Baxter AJ, Ferrari AJ, Erskine HE, et al. Global burden of disease attributable to mental and substance use disorders: findings from the Global Burden of Disease Study 2010. Lancet 2013; 382: 1575-86.

2 Layard R. The case for psychological treatment centres. BMJ 2006; 332: 1030-2.

3 Roth A, Fonagy P. What Works for Whom?: A Critical Review of Psychotherapy Research. Guilford Press, 2005.

4 National Institute for Health and Care Excellence. Depression in Adults: The Treatment and Management of Depression in Adults (Update - NICE Clinical guideline 90). NICE, 2009

5 Kaltenthaler E, Brazier J, De Nigris E, Tumur I, Ferriter M, Beverley C, et al. Computerised cognitive behaviour therapy for depression and anxiety update: a systematic review and economic evaluation. Health Technol Assess 2006; 10: 33

6 Andrews G, Cuijpers P, Craske MG, McEvoy P, Titov N. Computer therapy for the anxiety and depressive disorders is effective, acceptable and practical health care: a meta-analysis. PloS One 2010; 5: e13196.
7 Christensen H, Griffiths KM, Jorm AF. Delivering interventions for depression by using the internet: randomised controlled trial. BMJ 2004; 328: 265.

8 Gilbody S, Littlewood E, Hewitt C, Brierley G, Tharmanathan P, Araya R, et al. Computerised cognitive behaviour therapy (CCBT) as treatment for depression in primary care (REEACT trial): large scale pragmatic randomised controlled trial. BMJ 2015; 351: h5627.

9 Littlewood E, Duarte A, Hewitt C, Knowles S, Palmer S, Walker S, et al. A randomised controlled trial of computerised cognitive behaviour therapy for the treatment of depression in primary care: The Randomised Evaluation of the Effectiveness and Acceptability of Computerised Therapy (REEACT) trial. Health Technol Assess 2015; 19: viii, xxi-171.

10 Andersson G, Cuijpers P. Internet-based and other computerized psychological treatments for adult depression: a meta-analysis. Cog Behav Ther 2009; 38: 196-205.

11 Spek V, Cuijpers P, Nyklícek I, Riper H, Keyzer J, Pop V. Internet-based cognitive behaviour therapy for symptoms of depression and anxiety: a meta-analysis. Psychol Med 2007; 37: 319-28.

12 Waller R, Gilbody S. Barriers to the uptake of computerised cognitive behavioural therapy: a systematic review of the quantitative and qualitative evidence. Psychol Med 2008; 39: 705-12.

13 Cuijpers $\mathrm{P}$, Donker T, Johansson R, Mohr DC, van Straten A, Andersson G. Self-guided psychological treatment for depressive symptoms: a metaanalysis. Plos One 2011; 6: e21274.

14 Andersson G, Cuijpers P, Carlbring P, Riper H, Hedman E. Guided internetbased vs. face to face cognitive behavior therapy for psychiatric and somatic disorders: a systematic review and meta-analysis. World Psychiatry 2014; 13: 288-95.

15 Baumeister $\mathrm{H}$, Reichler L, Munzinger $\mathrm{M}$, Lin J. The impact of guidance on internet-based mental health interventions - a systematic review. Internet Interv 2014; 1: 205-15.

16 Kroenke K, Spitzer RL. The PHQ-9: a new depression and diagnostic severity measure. Psychiatr Ann 2002; 32: 509-21.

17 Moriarty AS, Gilbody S, McMillan D, Manea L. Screening and case finding for major depressive disorder using the Patient Health Questionnaire (PHQ-9): a meta-analysis. Gen Hosp Psychiatry 2015; 37: 567-76.

18 Spitzer RL, Kroenke K, Williams JBW, Lowe B. A brief measure for assessing generalized anxiety disorder: the GAD-7. Arch Intern Med 2006; 166: 1092-7.

19 Kroenke K, Spitzer RL, Williams JB. The PHQ-15: validity of a new measure for evaluating the severity of somatic symptoms. Psychosom Med 2002; 64: 258-66.

20 The EuroQol Group. EuroQOL - a new facility for the measurement of health related quality of life. Health Policy 1990; 16: 199-208

21 Chisholm D, Knapp MRJ, Knudsen HC, Amaddeo F, Gaite L, Van Wijngaarden B. Client Socio-Demographic and Service Receipt Inventory - European version: development of an instrument for international research EPSILON Study 5. Br J Psychiatry 2000; 177 (suppl 39): s28-33.

22 Brabyn S, Araya R, Barkham M, Bower P, Cooper C, Duarte A, et al. The second Randomised Evaluation of the effectiveness, cost-effectiveness and Acceptability of Computerised Therapy (REEACT-2) trial: does the provision of telephone support enhance the effectiveness of computer-delivered cognitive behaviour therapy? Health Technol Assess 2016; 20: 1-64.

23 Thorpe $K E$, Zwarenstein $M$, Oxman $A D$, Treweek S, Furberg $C D$, Altman DG, et al. A pragmatic-explanatory continuum indicator summary (PRECIS): a tool to help trial designers. J Clin Epidemiol 2009; 62: 464-75.

24 Cuijpers $P$, van Straten A, van Schaik A, Andersson G. Psychological treatment of depression in primary care: a meta-analysis. $\mathrm{Br} J \mathrm{Gen}$ Pract 2009; 59: e51-60.

25 Manea L, Gilbody S, McMillan D. Optimal cut-off score for diagnosing depression with the Patient Health Questionnaire (PHQ-9): a meta-analysis. Can Med Assoc J 2012; 184: E191-6.

26 Bower P, Kontopantelis E, Sutton A, Kendrick T, Richards DA, Gilbody S, et al. Influence of initial severity of depression on effectiveness of low intensity interventions: meta-analysis of individual patient data. BMJ 2012; 346: f540. 